\title{
Research and Development of Online Examination System
}

\author{
Zhao Qiao-fang \\ Department of Electrical and Mechanical Engineering \\ North China Institute of Science and Technology \\ Beijing, China \\ zhaoqiaofang@ncist.edu.cn
}

\author{
Li Yong-fei \\ Department of Computer \\ North China Institute of Science and Technology \\ Beijing, China \\ lyf518@ncist.edu.cn
}

\begin{abstract}
Examination System was necessary to separate teaching and testing. A web-based Examination System was developed with Java Web technologies. The system provided the functions, including question management, paper generation and test online. Also the combination of client-side programming and server-side programming techniques were used and analyzed.
\end{abstract}

Keywords-Examination System; JSP; Model1; JavaScript

\section{INTRODUCTION}

Examination System was helpful to separate teaching and testing. It promoted teacher to make the lesson planning and teach in the class carefully. It was also an important means of evaluating the teaching effect. Otherwise, it stimulated students to conscientiously study and attend a lecture, to do their homework independently. Therefore, the Examination System could improve the teaching level by providing better technical support.

Within the Examination System we developed, the teacher could set the score and number of different types of question according to their need. And then, the Examination System generated an exam paper randomly in accordance with the requirements. Finally, the paper in standard format and its answer could be outputted.

Taking into account the development of course content, the Examination System could easily update and add questions, to make the teaching content developing with the technology synchronously.

The Examination System also provided online testing capability for students. Students could log in the system at any time in the campus network, test themselves, understand their learning level, and adjust their learning progress.

\section{DESIGN OF ONLINE EXAMINATION SYSTEM}

\section{A. Model 1 in JSP}

Browser/Server model was an important network application development model. It was a special kind of Client/Server model, which used standard Web browser as Client-side, and Web Server as the Server-side. In Browser/Server model, the main business logic of program was implemented on the server side. Such application called Web application, had the advantages of good reusability and Easy maintenance [1]. Our Examination System was developed with JSP, and runs on the campus network. It was called online examination system.

JSP had become the main technology to create Web applications, because it was easy to master and could achieve rapid development. There were two types of building models JSP developing, respectively called Model 1 and Model 2. It was easily to combine with business logic (jsp:useBean), the server-side process(jsp:scriplet) and $\operatorname{HTML}(<\mathrm{html}>)$ in the JSP page. Therefore, it could be implemented to place display, business logic and process control in a JSP page, which result in rapid development of application. There was a large number of small-scale Web applications constituted by a group of JSP pages. The JSP-centric development model was called JSP model 1[2]. The architecture of JSP Model 1 was shown in Fig. 1.

\section{B. Architecture of Online Examination System}

Fig. 2 was the system structure of online examination system, which was designed based on Model 1. In the system, Web browser was used as client, JSP Engine as the business logic tier to achieve its function, and database system as the data layer.

(1) Client: Client was Web Browser, which implemented the system's display logic. The function was to send request to Web Server (including JSP Engine) through the Web browser by users (teachers or students). While Web Server return the requested HTML pages or HTML pages dynamically generated by JSP page to the client, which were shown in the Web browser.

2) Business Logic Tier: Business logic tier was achieved mainly by JSP and JavaBean running in the JSP Engine. It responded to client requests and achieved the business logic with the Web Server. Tomcat, an open source software, was used as the JSP Engine and Web Server.

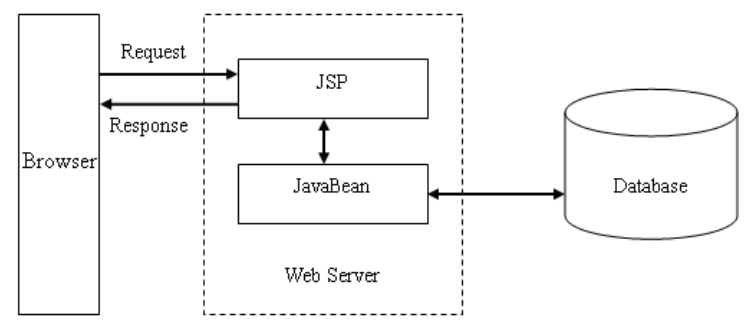

Figure 1. JSP model 1 architecture 


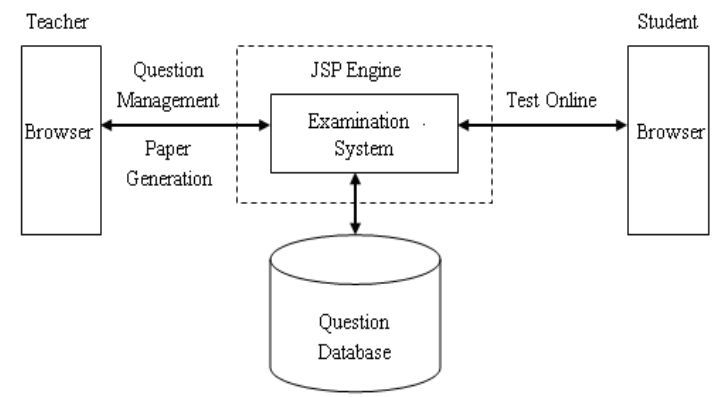

Figure 2. Architecture of online examination system

(3) Data Tier: Data tier was realized with database system, used to store the business data such as questions and papers and control data such as user data. MS ACCESS was used to achieve the data tier.

The JSP development model based on Model 1 is very suitable for quick and small scale application development.

\section{FUNCTIONS OF ONLINE EXAMINATION SYSTEM}

\section{A. Function Module of Online Examination System}

Fig. 3 was the function module diagram of online examination system. There were three modules in the system, including question management, paper generation and test online.

\section{B. Functions of Online Examination System}

1) Question Management: The functions of this module were querying, adding, deleting, and modifying the questions.

2) Paper Generating: The function of this module was randomly generating exam paper according to specified requirements. It was the core function of online examination system. The system randomly extracted questions from the question database in accordance with three restrictions, including question type, difficulty and chapter to generate an exam paper.

Here the term Paper Structure was a set of specified score and number of different types of question, which constituted a paper. There were five types of question, including false question, multiple choice, short answer question, cloze question and programming question. User could freely set a Paper Structure according to their needs. That meant to set how many types of questions used in a paper, how many questions and how many score for each type. The only restriction here was that the total score must be 100 points.

For example: A Paper Structure may include 10 false questions, each 1 point; 10 multiple choice questions, each 2 points; 2 short answer questions, each 5 points; 3 cloze questions, each 10 points and 2 programming questions, each 15 points.

The term Paper was a paper generated by extracting questions from the question database in accordance with a Paper Structure. A Paper consists of a sequence of questions, which formed the paper.

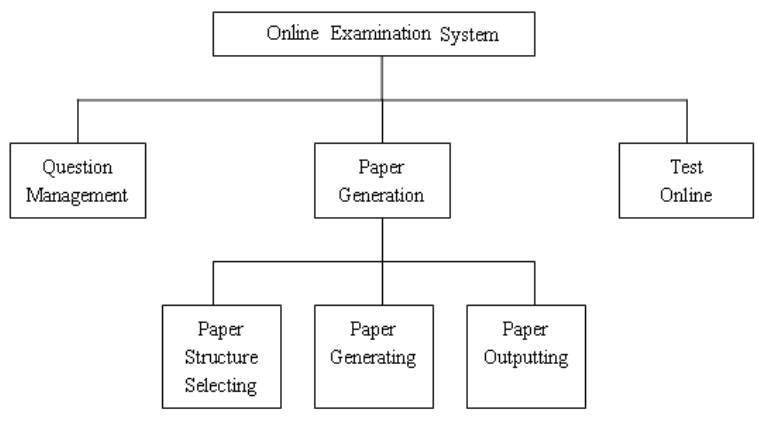

Figure 3. Function module diagram of online examination system

3) Test Online: Student user could use the function of test online to randomly select a paper or use a paper designated by teacher, and login the system at any time within the campus network, self-test online, and understand their learning level.

\section{Key TECHNOLOGY IN IMPLEMENTATION OF ONLINE EXAMINATION SYSTEM}

In test online module, the function on online answering was achieved with HTML forms in the answer page. Here it was necessary to implement five types of submit, including going to next question, going to previous question, going to specified question, going to rating and going to answering. These functions should be submitted to different pages, but there was a function to save the user's answer of current question that should be achieved in all submits. To do so, the form should be submitted to one page to save the user's answer, and then forward to corresponding next page. It was important to distinguish which button made submit when the server received.

To implement this feature, a technology combined with client-side JavaScript and server-side JSP was used. On the client side, a JavaScript function was binding with each submit button. Before submitting to server, the corresponding parameter was set to a certain value firstly. And on the server side, JSP technology was used to distinguish which made the submission according to the parameter value, and then make the appropriate process.

Here is sample code.

Client-side code:

$<$ script language $=$ "javascript ${ }^{\text {" }}>$

function examing()

\{

var next = document.getElementById("nextQNo"); next.setAttribute("value",999); return true;

\}

$</$ script $>$

$<$ form ...>

...

<input type="submit" value ="ANSWERING" onclick="return examing()"/>

$</$ form $>$ 


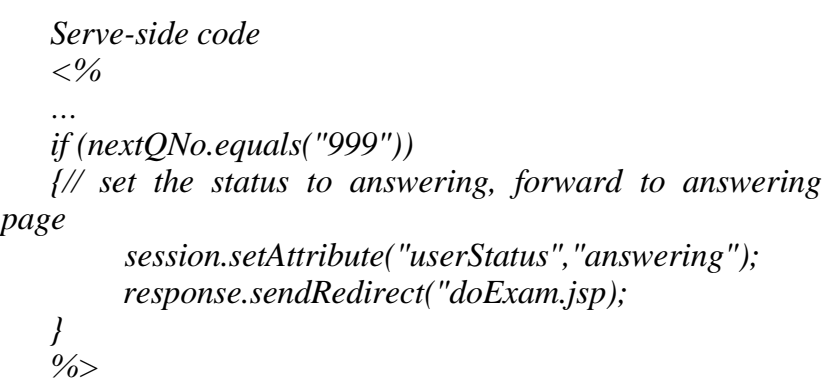

\section{SUMMARY}

JSP Model 1 development model was used to develop online examination system, with the combination of clientside and server side development technology. There are such functions as question management, paper generation and test online, which gave good aids for teachers to organize examination and students to study the course.

\section{REFERENCES}

[1] LIU Yang, GAO Lian-sheng and WANG Bin "Study and implement of distribution system based on J2EE and MVC design pattern" Computer engineering and Design(in Chinese). vol. 28, Apr. 2007, pp.1655-1658

[2] "JSP Architecture, JSP Model 1 architecture, JSP Model 2 architecture"

http://www.roseindia.net/tutorial/jsp/jsparchitecture.html

[3] FAN Ming-hu, SUN Bin "Desing and implementation of general test questions library mamagement system" Computer engineering and Design(in Chinese). vol. 28, May. 2007, pp.2185-2188

[4] LIU Li-ping, WANG Wen-jie "Design of Web-Based Exam-question with Self-study and Adaptive Adjusting" Computer System and Applications(in Chinese). Apr. 2006, pp.45-47 